\title{
Determination of seminal oxidation-reduction potential (ORP) as an easy and cost-effective clinical marker of male infertility
}

\author{
A. Agarwal, R. Henkel, R. Sharma, N. N. Tadros and E. Sabanegh
}

\section{Summary}

Oxidative stress (OS) is an important contributing factor to male infertility. While previous methods to measure seminal OS are time-consuming and limited to the use of freshly produced semen, oxidation reduction potential (ORP) is easier and quicker to perform and can also be used in frozen semen. Therefore, this study evaluated the clinical utility of ORP as a potential marker of male infertility. ORP was measured in semen samples from 293 patients and 15 fertile controls and categorised according to WHO criteria as normozoospermic, oligozoospermic, asthenozoospermic, teratozoospermic and oligoasthenoteratozoospermic. Receiver operating characteristic (ROC) curves were generated to differentiate these categories. Semen parameters were significantly different when subjects were grouped as control and patients or between the patient and normozoospermic group for concentration and morphology. ORP levels were significantly different between the control and normozoospermic group. When subjects were grouped based on concentration, motility, morphology or a combination of these, the area under the ROC curve, sensitivity, specificity, positive predictive value and cut-off values were significantly different. These differences were significant when combined with ORP and grouped with any two sperm abnormalities. In conclusion, ORP is a quick, easy, costeffective and reliable marker of semen quality as well as oxidative stress for use in a clinical setting.

\section{1 | INTRODUCTION}

Male infertility affects up to $12 \%$ of men globally (Agarwal, Ahmad, \& Sharma, 2015). Despite the ongoing controversies regarding the accuracy and predictive power of routine semen analysis, it continues to be used by many clinicians worldwide as a surrogate measure of a man's ability to father a child (Esteves, 2010). The American Urological Association (AUA) has proposed the inclusion of advanced tests of sperm function as complementary methods that can enhance the diagnostic accuracy of male infertility particularly in cases of unexplained infertility, one or more abnormal semen parameters, recurrent pregnancy loss or failure of intrauterine insemination (AUA, 2010).

Oxidative stress (OS) occurs when the oxidants overwhelm the ability of the available antioxidants to scavenge the increase in the production of reactive oxygen species (ROS); OS is a 
major contributor to male infertility. High levels of ROS are found in $25 \%-40 \%$ of infertile men and in 40\%-80\% of infertile patients with spinal cord injury (de Lamirande, Leduc, Iwasaki, Hassouna, \& Gagnon, 1995; Sharma \& Agarwal, 1996). In addition, infertile patients also have low levels of antioxidants in their seminal plasma (Ko, Sabanegh, \& Agarwal, 2014; Roychoudhury, Sharma, Sikka, \& Agarwal, 2016; Sharma, Pasqualotto, Nelson, Thomas, \& Agarwal, 1999). Yet, for successful fertilisation, a balance between oxidants and available antioxidants is essential for normal chromatin compaction in maturing spermatozoa during epididymal transit, capacitation, hyperactivation, acrosome reaction and sperm-oocyte fusion (Henkel et al., 2005; Ko et al., 2014; Sharma \& Agarwal, 1996).

Accurate assessment of OS can be important for the clinical diagnosis and management of male infertility, especially in those with unexplained and idiopathic infertility. However, currently available assays for OS are tedious and employ costly equipment and skills as well as large sample volumes. Furthermore, they measure only a known or a discrete quantity of oxidants (ROS by chemiluminescence assay), antioxidants (total antioxidant capacity [TAC] assay) or post hoc damage (MDA assay, sperm DNA fragmentation) (Agarwal et al., 2015; Henkel et al., 2005; Ko et al., 2014; Moazamian et al., 2015; Roychoudhury et al., 2016; Sharma \& Agarwal, 1996).

Oxidation-reduction potential or ORP is a direct measurement of oxidative stress and therefore the redox imbalance between oxidants and antioxidants in biological samples. It is an integrated measure of the balance between total oxidants (i.e., oxidised thiols, superoxide radicals, hydroxyl radicals, hydrogen peroxide, nitric oxide, peroxynitrite and transition metal ions) and total reductants (i.e., free thiols, ascorbate, $\alpha$-tocopherol, $\beta$-carotene and uric acid) (Agarwal, Sharma, Roychoudhury, du Plessis, \& Sabanegh, 2016; Agarwal et al., 2017).

ORP has been measured in a variety of biological specimens, including whole blood, serum, plasma and urine. As an indicator of oxidative stress, it has been shown to correlate well with illness and injury severity in trauma patients. For male infertility diagnostics, ORP represents a novel parameter that can be easily measured in fresh semen and seminal plasma up to $2 \mathrm{hr}$ after production, as well as in frozen samples with the MiOXSYS system (Agarwal, Sharma et al., 2016). Reference values of ORP have been established in infertile men and healthy men of proven and unproven fertility, and higher ORP measures are associated with a decrease in sperm concentration and motility. Using this parameter, we were able to identify oxidative stress as a possible factor in male infertility (Agarwal, Gupta, \& Sharma, 2016; Agarwal, Sharma et al., 2016; Agarwal et al., 2017).

As ORP may have a clinical utility as a potential marker of semen parameters, the objective of this study was to compare 1) semen parameters and ORP in normozoospermic men versus oligozoospermic, asthenozoospermic, teratozoospermic and oligoasthenoteratozoospermic men; 2) establish the cut-off value, sensitivity, specificity, positive predictive value (PPV) and negative predictive value (NPV) for semen parameters and ORP for these groups and 3) establish the sensitivity, specificity, PPV and cut-off for semen parameters and ORP in

\section{http://repository.uwc.ac.za}


subjects with two semen abnormalities to see whether ORP can serve as a single clinical marker of male infertility.

\section{2 | MATERIALS AND METHODS \\ 2.1 | Subjects}

The study was approved by the Institutional Review Board (IRB) of Cleveland Clinic. Semen samples were used from 15 controls with proven fertility and 293 infertile men referred to our andrology laboratory from August 2015 through October 2016. The infertile men attending our male infertility clinic had confirmed male factor infertility. All patients were evaluated by a male infertility specialist. All infertile patients were seeking treatment for male factor infertility (idiopathic infertility, varicoceles, infection and other known aetiologies). Patients with infection, azoospermia, severe oligozoospermia $\left(<1 \times 10^{6}\right.$ sperm $/ \mathrm{ml}$ ) or retrograde ejaculate were not included in the study. None of these patients were obese or had a history of chemotherapy or radiation. The female partners of these patients underwent a complete gynaecological investigation and were found to be healthy.

\section{2 | Study design}

After liquefaction, sperm count, motility, morphology and static oxidation-reduction potential (ORP) were determined in patients with adequate sperm counts. Semen samples were then categorised as oligozoospermic (OZ), asthenozoospermic (AZ) and teratozoospermic (TZ) according to the standard procedures described in 5 th edition of 2010 WHO guidelines [14] with the relevant cut-off values (OZ: sperm count $<15 \times 10^{6} / \mathrm{ml}$; AZ: total motility: <40\%; TZ: normal sperm morphology: <4\%). Subjects not falling into these categories were categorised as normozoospermic (NZ). These categories were compared with the two common categories "control" and "patient."

\section{3 | Semen analysis}

Following liquefaction, a manual semen analysis was performed according to World Health Organization, 2010 guidelines to determine sperm concentration and motility. Five microlitres of the sample was used for manual evaluation of motility using a MicroCell counting chamber (Vitrolife, San Diego, CA) with phase contrast optics set at $\times 20$ magnification (Agarwal et al., 2015).

\section{4 | Measurement of white blood cells}

Samples with a high concentration of round cells ( $>5$ per high power field) were examined for the presence of white blood cells, especially the granulocytes, by the peroxidase (Endtz) test (World Health Organization, 2010). To conduct the Endtz test, a 20- $\mu$ l well-mixed aliquot of the semen sample was mixed with one volume of PBS and 2 volumes of working Endtz solution in an amber coloured Eppendorf tube. After $5 \mathrm{~min}$, a drop of the aliquot was placed on a Makler chamber and examined for the presence of dark brown cells under an $\times 10$ bright field objective. Leukocytospermia was defined as the presence of $>1 \times 10^{6} \mathrm{WBC} / \mathrm{ml}$ according to the WHO criteria (World Health Organization, 2010). 


\section{5 | Measurement of oxidation-reduction potential (ORP)}

Immediately after semen liquefaction, ORP was evaluated using the MiOXSYS Analyzer (Aytu BioScience Inc., Englewood, CO) according to the method described previously (Agarwal, Roychoudhury, Bjugstad, \& Cho, 2016). In brief, $30 \mu \mathrm{l}$ of liquefied semen was applied to the MiOXSYS Sensor at room temperature and measured in triplicate.

The test starts when the sample fills the reference electrode, and the electrochemical circuit is completed. After a short period, the ORP values are displayed in millivolts $(\mathrm{mV})$ on the screen. Average values were recorded and normalised to sperm concentration to control for differences in cell numbers. Thus, data are presented as $\mathrm{mV} / 10^{6} \mathrm{sperm} / \mathrm{ml}$. The MiOXSYS System measures static ORP which provides a "snapshot" of the current balance of the redox system. A higher ORP level indicates an imbalance in the activity of all available oxidants relative to all available antioxidants in the seminal ejaculate and indicates a state of OS.

\section{6 | Statisticalanalysis}

Statistical analyses were carried out using the MedCalc Statistical Software, V. 17.1, [MedCalc Software bvba, Ostend, Belgium; https:// www.medcalc.org; 2017]. After checking for normal distribution of data with the Chi-squared test, the nonparametric tests, Spearman rank correlations, Mann-Whitney test and Jonckheere-Terpstra trend test were employed. In addition, Fisher's exact test and a receiver operating characteristic (ROC) curve analyses with subsequent Hanley and McNeil method for ROC comparison were used to further analyse the data. A $p$-value of $p<.05$ was considered significant.

\section{3 | RESULTS}

Summary statistics for all parameters taken are shown in Table 1. None of our controls presented with leukocytospermia. Among the patients, the incidence of leukocytosperrmia was 12.3\% (36/293). Of these, 7.2\% (21/293) had Endtz >1 × $10^{6} \mathrm{WBC} / \mathrm{ml} ; 3.1 \%(9 / 293)$ $>1$ but $<2 \times 10^{6} \mathrm{WBC} / \mathrm{ml}$; and $1.7 \%(6 / 293)$ presented with Endtz $>5 \times 10^{6} \mathrm{WBC} / \mathrm{ml}$ presented with leukocytospermia. Of the 293 patients, only 38 (13\%) were identified with clinical varicocele (38/293). A total of 308 subjects were enrolled in the study but due to low sperm concentration, not all subjects had motility, morphology and ORP performed. According to WHO criteria, 25.6\% (75/293) of the patients were categorised as "normozoospermic" compared to 53.3\% (8/15) among the fertile controls.

Semen parameters and ORP for OZ, AZ and TZ as well as "patients" and "controls" are shown in Table 2. Direct comparison of the different subject groups "control," "patient" and normozoospermia (NZ) revealed that except for ORP $(p=.2087)$, "patients" and "controls" differed significantly $(p<.05)$ for all parameters tested (Table 2). Generally, a trend for lower ORP values in subjects with higher sperm concentrations was found applying the Jonckheere-Terpstra trend test $(p<.0001)$. For the groups "patient" and NZ, significant differences were seen for all parameters. ORP was significantly lower in the control group ( $p<$ 
.0114) compared to the NZ group. No differences were seen in semen parameters between these two groups (Table 2).

Comparisons between the patient and oligozoospermic (OZ) group and asthenozoospermic (AZ) samples revealed significant differences $(p<.0032)$. For the teratozoospermic group (TZ), these differences were only significant for motility $(p=.0473)$ and morphology $(p<$ .0001). As expected, ORP was highly significant $(p<.0001)$ and negatively correlated with sperm concentration $(r=-0.840)$, motility $(r=-0.429)$ and weakly correlated with normal morphology $(r=-0.288)$.

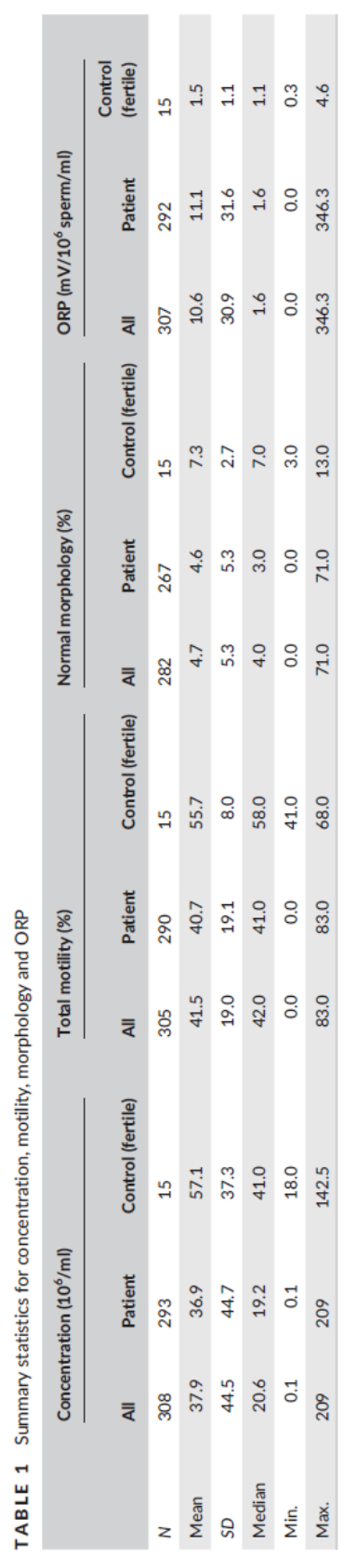


TAB LE 2 Comparison of concentration, motility, morphology and ORP between control and patient group; control and normozoospermic group and between patient and normozoospermic group

\begin{tabular}{|c|c|c|c|c|c|c|c|}
\hline Parameter & Control (Fertile) & Patient & NZ & $\mathrm{OZ}$ & $A Z$ & TZ & $p$-value \\
\hline Concentration $\left(10^{6} / \mathrm{ml}\right)$ & $\begin{array}{c}57.1 \pm 37.3 \\
(n=15)\end{array}$ & $\begin{array}{c}36.9 \pm 44.7 \\
(n=293)\end{array}$ & $\begin{array}{c}72.2 \pm 45.8 \\
(n=83)\end{array}$ & $\begin{array}{c}5.2 \pm 4.3 \\
(n=131)\end{array}$ & $\begin{array}{c}23.6 \pm 37.1 \\
(n=143)\end{array}$ & $\begin{array}{c}26.3 \pm 36.8 \\
(n=138)\end{array}$ & $\begin{array}{r}.0051^{\mathrm{a}} \\
.2325^{\mathrm{b}} \\
<.0001^{\mathrm{c}}\end{array}$ \\
\hline Total motility (\%) & $\begin{array}{c}55.7 \pm 8.0 \\
(n=15)\end{array}$ & $\begin{array}{c}40.7 \pm 19.1 \\
(n=290)\end{array}$ & $\begin{array}{c}57.9 \pm 11.3 \\
(n=83)\end{array}$ & $\begin{array}{c}30.1 \pm 16.0 \\
(n=128)\end{array}$ & $\begin{array}{c}25.0 \pm 11.8 \\
(n=143)\end{array}$ & $\begin{array}{c}37.1 \pm 17.8 \\
(n=138)\end{array}$ & $\begin{array}{r}.0001^{\mathrm{a}} \\
.4070^{\mathrm{b}} \\
<.0001^{\mathrm{c}}\end{array}$ \\
\hline Normal morphology (\%) & $\begin{array}{r}7.3 \pm 2.7 \\
(n=15)\end{array}$ & $\begin{array}{c}4.6 \pm 5.3 \\
(n=267)\end{array}$ & $\begin{array}{r}8.6 \pm 7.7 \\
(n=83)\end{array}$ & $\begin{aligned} 2.9 & \pm 2.5 \\
(n & =106)\end{aligned}$ & $\begin{aligned} 3.8 & \pm 6.7 \\
(n & =126)\end{aligned}$ & $\begin{array}{c}1.8 \pm 1.0 \\
(n=138)\end{array}$ & $\begin{array}{r}.0003^{\mathrm{a}} \\
.6907^{\mathrm{b}} \\
<.0001^{\mathrm{c}}\end{array}$ \\
\hline ORP $\left(\mathrm{mV} / 10^{6} \mathrm{sperm} / \mathrm{ml}\right)$ & $\begin{array}{r}1.5 \pm 1.3 \\
(n=15)\end{array}$ & $\begin{array}{c}11.1 \pm 31.6 \\
(n=292)\end{array}$ & $\begin{array}{r}1.2 \pm 3.1 \\
(n=83)\end{array}$ & $\begin{array}{c}23.3 \pm 44.5 \\
(n=130)\end{array}$ & $\begin{array}{c}13.9 \pm 29.6 \\
(n=143)\end{array}$ & $\begin{array}{r}7.6 \pm 12.6 \\
(n=137)\end{array}$ & $\begin{array}{r}.2087^{\mathrm{a}} \\
.0114^{\mathrm{b}} \\
<.0001^{\mathrm{c}}\end{array}$ \\
\hline
\end{tabular}

$\mathrm{NZ}$, normozoospermic; OZ, oligozoospermic; AZ, asthenozoospermic; TZ, teratozoospermic.

${ }^{\mathrm{a} C}$ Comparison between control (fertile men) and patient group.

${ }^{\mathrm{b}}$ Comparison between control (fertile men) and normozoospermic group.

'Comparison between patient and normozoospermic group. $p<.05$ was considered significant.

ROC curve analyses for the categories "patient"/"controls" revealed areas under the curve (AUC) for concentration (0.714; $p<.0001$ ), motility (0.752; $p<.0001)$ and morphology (0.776; $p$ <.0001) respectively. In contrast, ORP values for AUC were borderline significant between control and patients group $(0.596 ; p=.0400)$. The highest predictive power to distinguish between "patient" and "control" was for morphology (Table 3). However, if the classification is made for any two abnormalities (low sperm count [OZ], low motility [AZ], poor morphology [TZ] or non-specified) (Figure 1, Table 3), the ROC curve and the prediction of the semen abnormality became highly significant $(p<$.ooo1). ORP had high predictive power for OZ patients (AUC $=0.919$ ) (Table 3 ). Comparing the OAT and the non-OAT group, ORP the AUC and thus the predictive power of the test was not significantly different from those AUC's for motility and normal sperm morphology. Only the AUC for the sperm concentration (0.920) was significantly higher $(p=.0078)$ that that for ORP (0.822) (Table 3$)$.

\section{4 | DISCUSSION}

Many clinicians continue to rely on conventional semen parameters as a surrogate measure of a man's ability to father a child (Esteves, 2010). However, assessing male fertility potential based on semen analysis results is inaccurate due to (i) large inter- and intra-operator variation in conventional semen analysis as well as a large overlap in semen parameters reported between fertile and infertile men (Agarwal et al., 2006); (ii) semen characteristics that discriminate between infertile and fertile men are not well defined, and the end results fall within the accepted reference ranges in up to $40 \%$ of infertile men (Agarwal et al., 2006; Guzick et al., 2001; van der Steeg et al., 2011). This is because conventional semen analysis does not assess the changes occurring as a result of oxidative stress or DNA fragmentation (Duran, Morshedi, Taylor, \& Oehninger, 2002; Esteves, 2010; 3) reference values in the $5^{\text {th }}$ edition of WHO guidelines are derived from a population of fertile men that is not representative of the infertile population but rather of fertile men and the reference values set at fifth percentile distribution of the normal population which does not correlate with 
fecundity (Esteves, 2010) and 4) the WHO reference values are not globally representative (Esteves, 2010; Papillon-Smith, Baker, Agbo, \& Dahan, 2015). Almost 30\% of the infertile men who are unable to father a biological child present with a normal male infertility workup with one or two semen analysis (Esteves \& Agarwal, 2011). Therefore, supplementing conventional semen analysis with a reliable measure of sperm quality, such as oxidative stress, is recommended (Agarwal, Zini, \& Sigman, 2013).

Oxidative stress plays a central role in the pathogenesis of male infertility attributed to various aetiologies (Ko et al., 2014; Sharma \& Agarwal, 1996). It is a result of an imbalance between the reactive oxygen species (ROS) present in the ejaculate and the ability of the available antioxidants to quench these oxidants. Thus, excessive amounts of ROS have negative effects on sperm proteins, lipids and also lead to DNA fragmentation.

The direct measurement of ROS by chemiluminescence assay is the most widely utilised option with different methods being used (Agarwal et al., 2015; Agarwal, Roychoudhury et al., 2016), whereas measurement of antioxidant levels or activities using the TAC assay are less commonly used (Agarwal et al., 2015; Ko et al., 2014; Roychoudhury et al., 2016). 
TA B LE 3 ROC curve analysis for various parameters to differentiate between control (fertile) vs. patient and other groups and between subjects with two semen abnormalities

\begin{tabular}{|c|c|c|c|c|c|c|c|}
\hline Variable & AUC & Sensitivity (\%) & Specificity (\%) & PPV (\%) & NPV (\%) & Cut-off & $p$-value \\
\hline \multicolumn{8}{|c|}{ Control (Fertile) vs. Patient group } \\
\hline $\begin{array}{l}\text { Sperm concentration } \\
\left(10^{6} / \mathrm{ml}\right)\end{array}$ & 0.714 & 48.8 & 100 & 100 & 9.1 & $\leq 17.8$ & $<.0001$ \\
\hline Total motility (\%) & 0.752 & 71.4 & 80.0 & 98.6 & 12.6 & $<52$ & $<.0001$ \\
\hline Normal morphology (\%) & 0.776 & 51.3 & 93.3 & 99.3 & 9.7 & $\leq 3$ & $<.0001$ \\
\hline $\mathrm{ORP}\left(\mathrm{mV} / 10^{6}\right.$ sperm) & 0.596 & 40.4 & 93.3 & 99.2 & 7.4 & $>2.63$ & .0400 \\
\hline \multicolumn{8}{|c|}{ Oligozoospermic vs. non-oligozoospermic group } \\
\hline \multicolumn{8}{|c|}{$\begin{array}{l}\text { Sperm concentration } \\
\left(10^{6} / \mathrm{ml}\right)\end{array}$} \\
\hline Total motility (\%) & 0.801 & 68.8 & 75.7 & 67.2 & 77.0 & $\leq 38$ & $<.0001$ \\
\hline Normal morphology (\%) & 0.721 & 70.8 & 64.2 & 54.3 & 78.5 & $\leq 3$ & $<.0001$ \\
\hline ORP $\left(\mathrm{mV} / 10^{6}\right.$ sperm) & 0.919 & 81.5 & 92.7 & 89.1 & 87.2 & $>2.63$ & $<.0001$ \\
\hline \multicolumn{8}{|c|}{ Asthenozoospermic vs. non-asthenozoospermic group } \\
\hline $\begin{array}{l}\text { Sperm concentration } \\
\left(10^{6} / \mathrm{ml}\right)\end{array}$ & 0.733 & 62.9 & 80.3 & 73.8 & 71.0 & $\leq 13.3$ & $<.0001$ \\
\hline Total motility (\%) & - & - & - & - & - & - & - \\
\hline Normal morphology (\%) & 0.691 & 65.9 & 64.7 & 60.1 & 70.1 & $\leq 3$ & $<.0001$ \\
\hline $\mathrm{ORP}\left(\mathrm{mV} / 10^{6}\right.$ sperm) & 0.685 & 46.9 & 86.3 & 75.3 & 64.7 & $>5.2$ & $<.0001$ \\
\hline \multicolumn{8}{|c|}{ Teratozoospermic vs. non-teratozoospermic group } \\
\hline $\begin{array}{l}\text { Sperm concentration } \\
\left(10^{6} / \mathrm{ml}\right)\end{array}$ & 0.725 & 69.6 & 68.1 & 67.6 & 70.0 & $\leq 24.8$ & $<.0001$ \\
\hline Total motility (\%) & 0.676 & 60.1 & 70.1 & 65.9 & 64.7 & $\leq 40$ & $<.0001$ \\
\hline Normal morphology (\%) & - & - & - & - & - & - & - \\
\hline ORP $\left(\mathrm{mV} / 10^{6}\right.$ sperm) & 0.655 & 65.7 & 65.3 & 64.3 & 66.7 & $>1.4$ & $<.0001$ \\
\hline \multicolumn{8}{|c|}{ Subjects with any two semen abnormalities } \\
\hline $\begin{array}{l}\text { Sperm concentration } \\
\left(10^{6} / \mathrm{ml}\right)\end{array}$ & 0.877 & 80.9 & 89.9 & 86.2 & 85.8 & $\leq 14.9$ & $<.0001$ \\
\hline Total motility (\%) & 0.903 & 88.6 & 84.5 & 81.7 & 90.4 & $\leq 40$ & $<.0001$ \\
\hline Normal morphology (\%) & 0.837 & 85.2 & 75.9 & 71.0 & 88.1 & $\leq 3$ & $<.0001$ \\
\hline $\mathrm{ORP}\left(\mathrm{mV} / 10^{6}\right.$ sperm $)$ & 0.803 & 64.6 & 83.9 & 75.7 & 75.4 & $>2.7$ & $<.0001$ \\
\hline \multicolumn{8}{|c|}{ Oligoasthenoteratozoospermic vs. non-oligoasthenozoospermic group } \\
\hline $\begin{array}{l}\text { Sperm concentration } \\
\left(10^{6} / \mathrm{ml}\right)\end{array}$ & 0.920 & 98.3 & 80.1 & 57.0 & 99.5 & $\leq 13.3$ & $<.0001$ \\
\hline Total motility (\%) & 0.894 & 94.8 & 75.9 & 50.5 & 98.3 & $\leq 37$ & $<.0001$ \\
\hline Normal morphology (\%) & 0.841 & 100 & 64.3 & 42.0 & 100 & $\leq 3$ & $<.0001$ \\
\hline ORP (mV/10 6 sperm) & 0.822 & 69.0 & 87.4 & 58.8 & 91.5 & $>5.3$ & $<.0001$ \\
\hline
\end{tabular}

AUC, area under curve; PPV, positive predictive value; NPV, negative predictive value.

Previously, we used ROS levels to compare semen parameters and pregnancy in fertile men and had established a pregnancy compared to infertile men (Agarwal, Sharma et al., 2014). Infertile men had consistently poor semen parameters that correlated negatively with ROS levels irrespective of the clinical diagnosis. Similarly, when fertile donors were compared with different combinations of oligozoospermia and teratozoospermia, the ROS cut-off in the control group was significantly lower with a higher sensitivity and specificity when compared to different combinations of oligozoospermia and teratozoospermia. A positive relationship was seen between poor semen parameters and elevated ROS levels suggesting an underlying mechanism in these infertile patients (Agarwal, Mulgund, Sharma, \& Sabanegh, 2014; Agarwal, Tvrda, \& Sharma, 2014). 
More recently, we demonstrated that the ORP measurement by the MiOXSYS system is an invaluable clinical tool that simplifies complex OS assays. Higher ORP values indicate an imbalance in the activity of oxidants relative to antioxidants. Using ORP as a measure of oxidative stress (Agarwal, Sharma et al., 2016; Agarwal et al., 2017), our data reinforce the impression that oxidative stress is related to poor semen quality (Agarwal, Mulgund et al., 2014; Agarwal, Tvrda et al., 2014; Ko et al., 2014; Sharma et al., 1999). ORP measured in semen samples from male partners of infertile couples suggests that ORP is significantly lower in those samples that exhibit normal semen parameters.

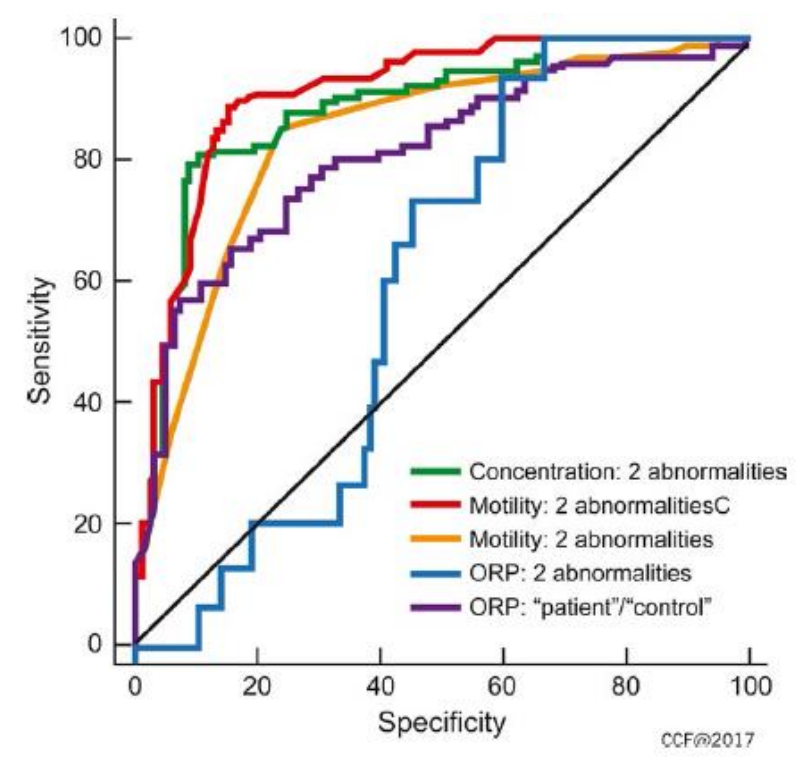

FIGURE 1 Receiver operating curve showing sensitivity and specificity for sperm parameters with two abnormalities alone and ORP combined with two semen abnormalities as well as ORP in patients and controls

In an earlier study examining ORP data from 218 normozoospermic men and 69 OAT patients, higher levels of oxidative stress were recorded in oligoasthenoteratozoospermic group ( $p$.0001) and confirmed by ORP (Agarwal, Gupta et al., 2016). Higher ORP values were recorded in these men than the normozoospermic men who had normal sperm concentration, motility and morphology are supported by previous finding by Cavallini (2006).

In an earlier study, an ORP cut-off value of $1.36 \mathrm{mV} / 10^{6} \mathrm{sperm} / \mathrm{ml}$ was capable of predicting abnormality in semen quality with a sensitivity of $69.6 \%$, a specificity of $83.1 \%$, positive predictive value of $85.3 \%$ and a negative predictive value (NPV) of $65.9 \%$. The accuracy of the test was $75.2 \%(A U C=0.770)$. Negative correlations were reported in this study with sperm concentration, total sperm count, motility and morphology. Furthermore, the intra-observer and inter-observer reliability of ORP were very strong (correlations $>0.97 \%$ ), suggesting that ORP testing can be clinically useful in identifying OS in men at risk of infertility that would otherwise go undetected with a routine semen analysis (Agarwal et al., 2017). 
In the present study, an larger number of subjects were enroled and examined for sperm concentration, motility, normal sperm morphology and ORP. The subjects were categorised as normozoospermic, oligozoospermic, asthenozoospermic, teratozoospermic or oliogoasthenoteratozoospermic. As expected, significant differences in semen parameters between the control and patient group were found. The distribution of leukocytospermia among the patients of $12.2 \%$ is similar $(12 \%-19 \%)$ to that reported in the literature (Henkel et al., 2005; Sharma \& Agarwal, 1996; Tremellen, 2008; World Health Organization, 2010). More importantly, the ORP levels were also significantly higher in all subgroups (when compared with the normozoospermic group (Table 2). ORP was able to distinguish between the patient and normozoospermic group $(p<.0001)$, even when a patient presented with normal semen parameters (NZ).

When the subjects were grouped based on semen parameters into oligozoospermic versus non-oligozoospermic, asthenozoospermic versus non-asthenozoospermic or teratozoospermic versus nonteratozoospermic; both sperm parameters and ORP were significantly different $(p<.0001)$ (Table 3$)$.

A similar trend in significant differences in ROC parameters was seen between the oligoasthenoteratozoospermic versus non-oligoasthenoteratozoospermic group for both sperm parameters and ORP. Semen samples from oligoasthenoteratozoospermic (OAT) patients have higher levels of isoprostane (a by-product of arachidonic acid peroxidation), lower levels of catalase and have lower TAC levels and may contribute to the elevated ORP (Khosrowbeygi \& Zarghami, 2007).

To further establish the clinical utility of ORP, we examined the sensitivity and specificity of ORP in control and patients as well as in semen samples with at least 2 semen abnormalities (Table 3; Figure 1). The AUC was significantly higher when the ROC curve was calculated for ORP in patients with oligozoospermia or two abnormal semen parameters compared to one abnormal semen parameters only.

For any diagnostic test to be considered valid for clinical use, it should combine high sensitivity, specificity, and positive and negative predictive values. When a given test has a high sensitivity, it will correctly identify subjects with the condition of interest, that is infertility as in this case. In contrast, highly specific tests correctly identify subjects who do not have this condition of interest. Although predictive values are influenced by sensitivity and specificity, they depend on the prevalence of the condition of interest in the general population.

The classification of ejaculates according to the WHO criteria "normozoospermia," "asthenozoospermia," "oligozoospermia," "teratozoospermia" etc. as well as the classification into "patient" and "donor" is standard practice in clinic and research and is regularly used to distinguish between different subjects. Therefore, it is important to notice that only about half of the "fertile" donors could be classified as being normozoospermic. For the overall study group, this percentage was about one-fourth. In the light of doing a semen analysis 
including sperm count, motility and normal sperm morphology in a clinical laboratory in an andrology unit or even in the embryology laboratory just before an assisted reproduction procedure, carrying out these tests is time-consuming and costly. If one calculates the actual cost (consumables and technical charges only as the billing charges for these tests could vary greatly between the providers) for a routine semen analysis with sperm count, motility and normal morphology (World Health Organization, 2010) is about $\$ 35.00$, the cost to measure ORP is approximately $\$ 10.00$ or one-third the cost for a normal semen analysis. More importantly for the patient, measurement of ORP takes only about 5 min and can therefore be taken while the patient is still visiting the specialist. Results can therefore be discussed immediately without the need for a second visit. Hence, determination of ORP is easy, quick and cost-effective, with good predictive power, and the test can be of assistance to guide the clinician and embryologist in their decisions.

In a recent publication examining the relationship between ORP and semen quality as well as examining the changes in sperm parameters over time, at a cut-off of $1.57 \mathrm{mV} / 10^{6} \mathrm{sperm}$, we found high sensitivity of $70.4 \%$ and specificity of $88.1 \%$ with a PPV of $95.5 \%$, irrespective of their clinical diagnoses (Agarwal \& Wang, 2017). At a cut-off of $2.59 \mathrm{mV} / 10^{6}$ sperm, ORP could differentiate controls, from infertile men suffering from oligozoospermia. This finding also points out to the fact that OS may be a primary mechanism in reducing sperm concentration in these patients.

Our results demonstrate a number of important findings; (I) semen parameters and ORP both show significant differences in the ROC curves for AUC, sensitivity, specificity and predictive value especially when abnormal semen parameters (concentration, motility or morphology are compared to those with normal semen parameters or when subjects with two of the three abnormal semen parameters are compared); (ii) semen analysis requires highly trained staff, is costly and does not test for sperm function characteristics such as OS; (iii) ORP represents a direct measure of oxidative stress that is objective, repeatable and can be performed in less than 4 min without costly equipment or highly trained staff. Therefore, ORP represents an effective and more cost-efficient measure of OS in comparison with ROS, TAC and MDA assays. The test can be performed while the patient is still with the physician, and therefore, the result can be discussed immediately. This saves the patient from returning for a second time to discuss the test results.

The large variation in the distribution of ORP levels between the controls and the different subgroups signifies the importance of OS in the pathophysiology of male infertility. When controls with unproven fertility were included in the control group analysis, no differences were seen in the ROC curve. Therefore, it is important to determine the fertility status in patients and controls as not every control might be fertile and not every patient infertile based only on the semen parameters. This is even valid for the distinction of subjects according to WHO criteria. Men with infertility had a significantly higher ORP value in all these subgroups than men in the control group. Assessing ORP in men seeking fertility should help the clinician identify an important sperm function characteristic that would otherwise go undetected during the semen analysis and aid decision-making process. It can 
also be used to monitor the effectiveness of treatment for oxidative stress. The main limitation of the present study was that the samples were taken from a male infertility clinic, and therefore, we are unable to determine further outcome studies associated with clinical pregnancy and live birth outcomes. Another limitation of our study was the small number of fertile controls. However, our analysis shows statistical significance despite the small size of the control group.

In conclusion, our results demonstrate that measurement of ORP is an accurate and rapid test of semen quality that can be used routinely in clinical practice. The test is not a replacement for semen analysis, rather it can be used in conjunction with a routine semen analysis to help evaluate oxidative stress induced male infertility. Due to the rapid turnaround time and ease of use, it can be potentially used as a stand-alone sperm function test to help rule in male infertility cases associated with oxidative stress when semen analysis is not readily available.

\section{ACKNOWLEDGEMENTS}

The study was supported by funds from the American Center for Reproductive Medicine. The authors are grateful to the Andrology Center technologists for scheduling the study subjects.

\section{ORCID}

A.Agarwal http://orcid.org/oooo-0003-0585-1026

R. Henkel http://orcid.org/oooo-0003-1128-2982

N.N.Tadros http://orcid.org/oooo-0oo2-2272-235X

R. Sharma http://orcid.org/oooo-0oo2-9664-6978 


\section{REFERENCES}

Agarwal, A., Ahmad, G., \& Sharma, R. (2015). Reference values of reactive oxygen species in seminal ejaculates using chemiluminescence assay. Journal of Assisted Reproduction and Genetics, 32, 1721-1729.

Agarwal, A., Gupta, S., \& Sharma, R. (2016). Oxidation-Reduction potential measurement in ejaculated semen samples. In Agarwal A., Gupta S., Sharma R. (eds) Andrological evaluation of male infertility: A laboratory guide, Vol. 22 (pp. 165-170). Switzerland: Springer Nature, Springer International Publishing, AG.

Agarwal, A., Mulgund, A., Sharma, R., \& Sabanegh, E. (2014). Mechanisms of oligozoospermia: An oxidative stress perspective. Systems Biology in Reproductive Medicine, 6o, 206-216.

Agarwal, A., Roychoudhury, S., Bjugstad, K. B., \& Cho, C.-L. (2016). Oxidation-reduction potential of semen: What is its role in the treatment of male infertility? Therapeutic Advances in Urology, 8, 302-318.

Agarwal, A., Roychoudhury, S., Sharma, R., Gupta, S., Majzoub, A., \& Sabanegh, E. (2017). Diagnostic application of oxidation-reduction potential assay for measurement of oxidative stress: Clinical utility in male factor infertility. Reproductive Biomedicine Online, 34, 48-57.

Agarwal, A., Sharma, R. K., Nallella, K. P., Thomas, A. J. Jr, Alvarez, J. G., \& Sikka, S. C. (2006). Reactive oxygen species as an independent marker of male factor infertility. Fertility and Sterility, 86, 878-885.

Agarwal, A., Sharma, R., Roychoudhury, S., du Plessis, S., \& Sabanegh, E. (2016). MiOXSYS: A novel method of measuring oxidation reduction potential in semen and seminal plasma. Fertility and Sterility, 106, 566-573.

Agarwal, A., Sharma, R. K., Sharma, R., Assidi, M., Abuzenadah, A. M., Alshahrani, S., ... Sabanegh, E. (2014). Characterizing semen parameters and their association with reactive oxygen species in infertile men. Reproductive Biology and Endocrinology, 12, 33 .

Agarwal, A., Tvrda, E., \& Sharma, R. (2014). Relationship amongst teratozoospermia, seminal oxidative stress and male infertility. Reproductive Biology and Endocrinology, 12, 45.

Agarwal, A., \& Wang, S. M. (2017). Clinical relevance of oxidation-reduction potential in the evaluation of male infertility. Urology, 2017(104), 84-89.

Agarwal, A., Zini, A., \& Sigman, M. (2013). Is sperm DNA integrity assessment useful? Journal of Urology, 19o, 1645-1647.

AUA (2010). The Optimal Evaluation of the Infertile Male. AUA Best Practice Statement. Linthicum, MD: AUA.

Cavallini, G. (2006). Male idiopathic oligoasthenoteratozoospermia. Asian Journal of Andrology, 8, 143-157.

Duran, E. H., Morshedi, M., Taylor, S., \& Oehninger, S. (2002). Sperm DNA quality predicts intrauterine insemination outcome: A prospective cohort study. Human Reproduction, $17,3122-3128$.

Esteves, S. C. (2010). Clinical relevance of routine semen analysis and controversies surrounding the 2010 World Health Organization criteria for semen examination. International Brazilian Journal of Urology, 40, 443-453.

\section{http://repository.uwc.ac.za}


Esteves, S. C., \& Agarwal, A. (2011). Novel concepts in male infertility. International Brazilian Journal of Urology, 37, 5-15.

Guzick, D. S., Overstreet, J. W., Factor-Litvak, P., Brazil, C. K., Nakajima,

S. T., Coutifaris, C., ... Network National Cooperative Reproductive Medicine. (2001). Sperm morphology, motility, and concentration in fertile and infertile men. New England Journal of Medicine, 345, 1388-1393.

Henkel, R., Kierspel, E., Stalf, T., Mehnert, C., Menkveld, R., Tinneberg,

H. R., ... Kruger, T. F. (2005). Effect of reactive oxygen species produced by spermatozoa and leukocytes on sperm functions in non-leukocytospermic patients. Fertility and Sterility, 83, 635-642.

Khosrowbeygi, A., \& Zarghami, N. (2007). Levels of oxidative stress biomarkers in seminal plasma and their relationship with seminal parameters. BMCClinical Pathology, 7, 6 .

Ko, E. Y., Sabanegh, E. S. Jr, \& Agarwal, A. (2014). Male infertility testing: Reactive oxygen species and antioxidant capacity. Fertility and Sterility, 102, 1518-1527.

de Lamirande, E., Leduc, B. E., Iwasaki, A., Hassouna, M., \& Gagnon, C. (1995). Increased reactive oxygen species formation in semen of patients with spinal cord injury. Fertility and Sterility, 63, 637-642.

Moazamian, R., Polhemus, A., Connaughton, H., Fraser, B., Whiting, S., Gharagozloo, P., \& Aitken, R. J. (2015). Oxidative stress and human spermatozoa: Diagnostic and functional significance of aldehydes generated as a result of lipid peroxidation. Molecular Human Reproduction, 21, 502-515.

Papillon-Smith, J., Baker, S. E., Agbo, C., \& Dahan, M. H. (2015). Pregnancy rates with intrauterine insemination: Comparing 1999 and 2010 World Health Organization semen analysis norms. Reproductive Biomedicine Online, 30, 392-400.

Roychoudhury, S., Sharma, R., Sikka, S., \& Agarwal, A. (2016). Diagnostic application of total antioxidant capacity in seminal plasma to assess oxidative stress in male factor infertility. Journal of Assisted Reproduction and Genetics, 33, 627-635.

Sharma, R. K., \& Agarwal, A. (1996). Role of reactive oxygen species in male infertility. Urology, 48, 835-850.

Sharma, R. K., Pasqualotto, F. F., Nelson, D. R., Thomas, A. J. Jr, \& Agarwal, A. (1999). The reactive oxygen species-total antioxidant capacity score is a new measure of oxidative stress to predict male infertility. Human Reproduction, 14, 2801-2807.

van der Steeg, J.W., Steures, P., Eijkemans, M.J., Habbema, J.D., Hompes, P.G., Kremer, J.A., ... Collaborative Effort for Clinical Evaluation in Reproductive Medicine Study Group. (2011). Role of semen analysis in subfertile couples. Fertility and Sterility. 95, 10131019.

Tremellen, K. (2008). Oxidative stress and male infertility-a clinical perspective. Human Reproduction Update, 14, 243-258.

World Health Organization (2010). WHO Laboratory Manual for the Examination and Processing of Human Semen, 5th ed.. Geneva, Switzerland: World Health Organization. 